CENTRO UNIVERSITÁRIO INTA - UNINTA

RESIDÊNCIA MULTIPROFISSIONAL EM URGÊNCIA E EMERGÊNCIA

SANTA CASA DE MISERICÓRDIA DE SOBRAL

e24261

CONSTRUÇÃO DO PROTOCOLO ASSISTENCIAL AO PACIENTE SÉPTICO EM UMA UNIDADE DE EMERGÊNCIA

SAMUEL DE SOUSA OLIVEIRA

SOBRAL - CE 
SAMUEL DE SOUSA OLIVEIRA

CONSTRUÇÃO DO PROTOCOLO ASSISTENCIAL AO PACIENTE SÉPTICO EM UMA UNIDADE DE EMERGÊNCIA

$\begin{aligned} & \text { Trabalho de Conclusão de Residência } \\ & \text { apresentando a } \\ & \text { Multiprofissional em Urgência e }\end{aligned}$
Emergência da Santa Casa de
Misericórdia de Sobral/UNINTA como
requisito para obtenção do título de
Especialista em Urgência e Emergência
com caráter de Residência.
Orientador: Dr. Tiago Sousa de Melo

SOBRAL - CE 
SAMUEL DE SOUSA OLIVEIRA

\section{CONSTRUÇÃO DO PROTOCOLO ASSISTENCIAL AO PACIENTE SÉPTICO EM UMA UNIDADE DE EMERGÊNCIA}

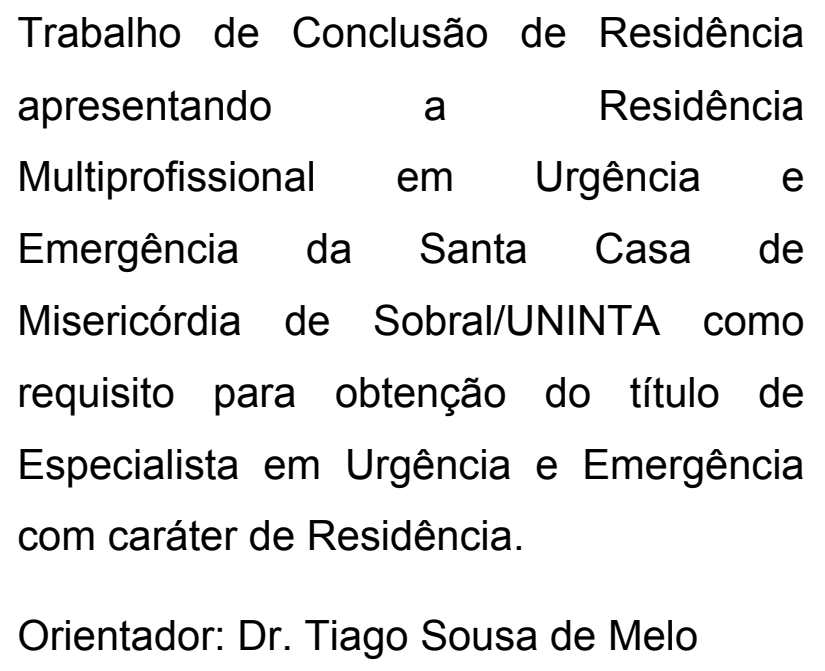

Aprovada em:

BANCA EXAMINADORA

Dr. Tiago Sousa de Melo

Coordenador geral do programa de residência orientador do trabalho

Prof. Mestre Denis Frota Guimarães

Hobber Kildare Sousa Silva

Coordenador da Emergência

Santa Casa de Misericórdia de Sobral 
A Deus, que se mostrou criador, que foi criativo. Seu fôlego de vida em mim me foi sustento e me deu coragem para questionar realidades e propor sempre um novo mundo de possibilidades. 


\section{AGRADECIMENTOS}

Primeiramente agradeço ao dono de tudo, que fez todas as coisas, dono de todos os seres viventes, tudo é dEle, tudo é pra Ele ao único que é digno de toda honra, de toda glória, de todo louvor e adoração, o meu Senhor e Salvador JESUS CRISTO. Sem Ele nada disso seria possível, sem ele eu não teria chegado aqui. Ah! JESUS graças te dou por onde permitiu eu chegar, pelo que me permitiu fazer, pelo que eu me tornei e pelo que ainda serei.

Agradecer a minha mãe Tânia Sousa pelo amor que me deu, pelos ensinamentos, por me ensinar a batalhar e ter honestidade nas minhas conquistas. Agradeço pelo apoio e por investir nos meus maiores sonhos. Jamais imaginei um dia ser residente e hoje finalizo esse sonho devido seu trabalho, esforço e perseverança por acreditar em dias melhores para nós.

Não há palavras nesse mundo nem ações que eu possa fazer para expressar o meu mais profundo amor e agradecimento àquela que ama acima de tudo sem eu mesmo merecer. Devo a ti essa conquista, essa vitória, essa realização profissional, enfim minha mãe, eu chego ao final dessa caminhada e pronto para encarar outras que mais vierem pela frente.

Meus amigos, em especial aqueles que estiveram comigo em todos os momentos. Aqueles que torceram, rezaram e comemoraram junto a mim, todas as minhas conquistas durante esses anos, lembrarei-me de todos com carinho e admiração. Todos que cada um do seu jeito marcou a minha vida se fez especial e são muitos importantes. São poucos, mas verdadeiros.

Agradeço ao meu orientador Prof Dr. Tiago Melo por gentilmente ter me ajudado, guiado no decorrer deste trabalho me oferecendo todo o suporte necessário. Um exemplo como pessoa e profissional.

E por fim, queria agradecer a eu mesmo, por ter seguido nessa caminhada. Sigo firme, pois sou como a haste fina, que qualquer brisa verga, nenhuma espada corta. 

"Tudo tem o seu tempo determinado, e há tempo para todo o propósito debaixo do céu."

Eclesiastes 3:1 


\section{LISTA DE ABREVIATURAS E SIGLAS}

ANVISA

CEP

CNS

EP

IRAS

MS

TCLE

UTI

ILAS

SIRS

SSC

Sepse
Agência Nacional de Vigilância Sanitária

Comitê de Ética em Pesquisa

Conselho Nacional de Saúde

Educação Permanente

Infecção Relacionado á Assistência á Saúde

Ministério da saúde

Termo de Consentimento Livre e Esclarecido

Unidade de Terapia Intensiva

Instituto Latino Americano de Sepse

Síndrome de resposta inflamatória sistêmica

Surviving Sepsis Campaing/Campanha de Sobrevivência a 


\section{SUMÁRIO}

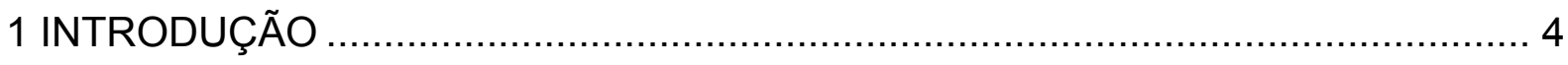

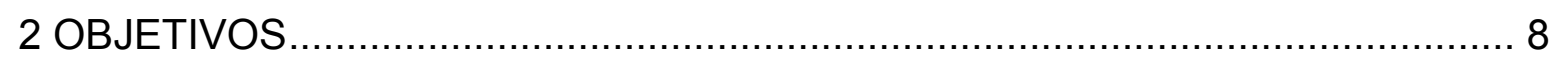

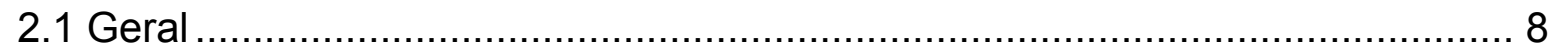

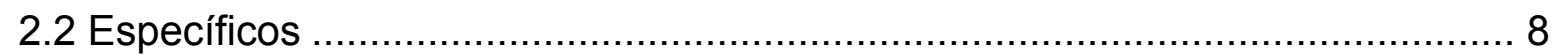

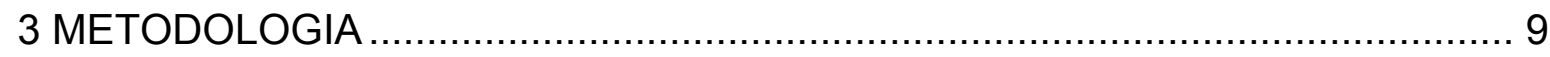

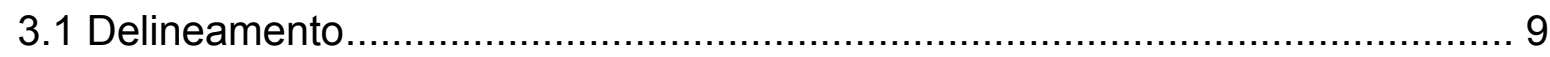

3.2 Local e período da pesquisa.................................................................. 10

3.3 Definição do problema................................................................................ 10

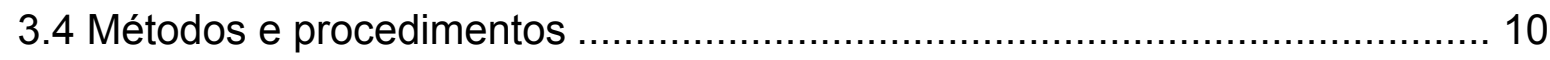

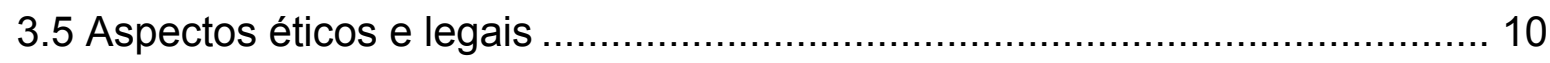

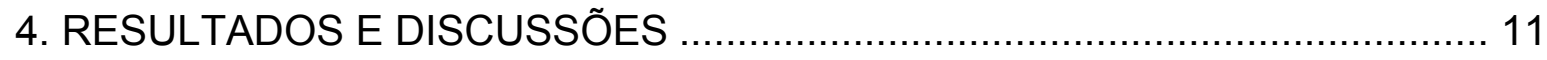

4.1 Etapas para a construção do protocolo assistencial ..................................... 11

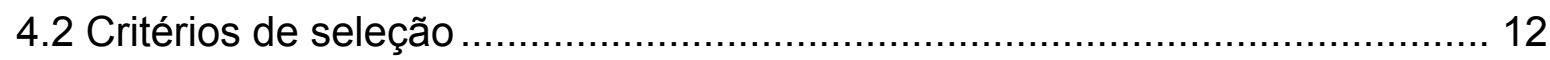

4.3 A Construção do protocolo sepse ............................................................. 13

4.4 Construção da ficha de triagem e fluxograma para pacientes com suspeita de

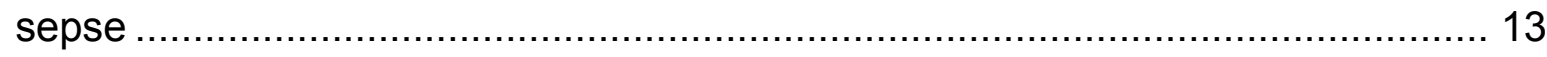

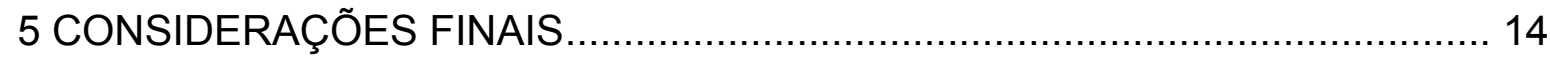

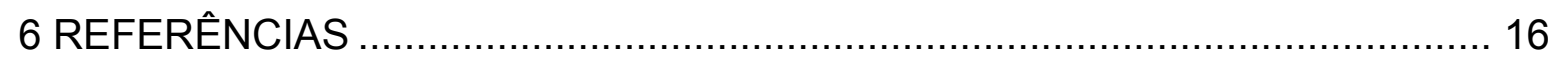

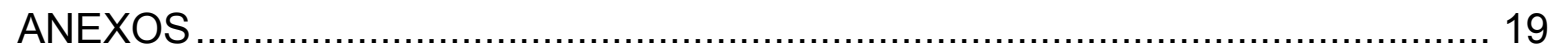

ANEXO A - Protocolo de atendimento - Pacotes de 1 hora e de 6 horas............ 20

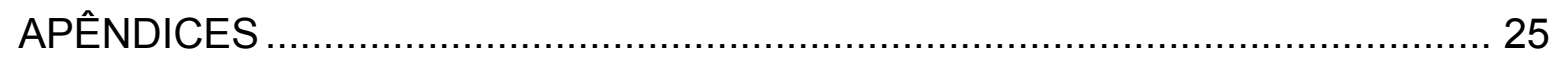

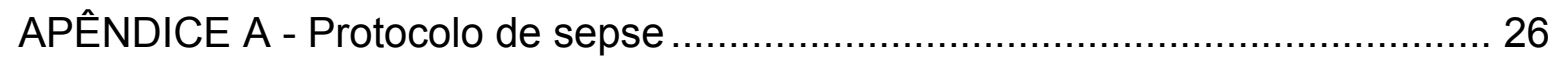

APÊNDICE B - FLUXOGRAMA DE ATENDIMENTO PARA PACIENTES COM

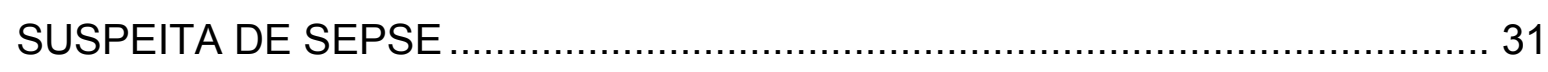

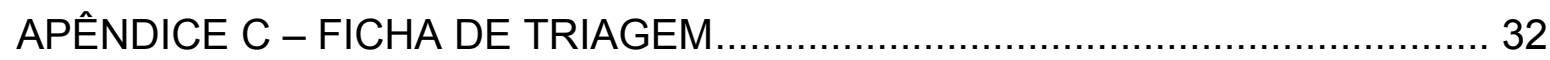




\section{INTRODUÇÃO}

A palavra sepse foi usada por Hipócrates em seus escritos datados de 400 anos antes de Cristo. Deriva do grego, tendo por significado decomposição de animal, vegetal ou substância orgânica na presença de bactéria. Acreditava-se na época que essa decomposição acontecia no cólon e liberava "substâncias perigosas" que poderiam causar sepse (FUNK; PARRILLO; KUMAR, 2009).

A sepse é uma das doenças mais desafiadoras da medicina. Têm sido dispendidos esforços consideráveis para um melhor entendimento da inflamação sistêmica que caracteriza essa síndrome. Apesar de sua importância, seu reconhecimento, muitas vezes, ainda não ocorre em tempo hábil, deixando margem para a ocorrência de disfunção de múltiplos órgãos e sistemas. Seu manejo sofreu profundas mudanças na última década, havendo hoje inúmeras orientações com base em evidências advindas de estudos no cenário clínico (HENKIN et al., 2009).

A sepse é um conjunto de manifestações graves em todo o organismo, sendo produzida por uma infecção. A sepse era conhecida antigamente como septicemia ou infecção no sangue. Hoje, é mais conhecida como infecção generalizada. $\mathrm{Na}$ verdade, não é a infecção que está em todos os locais do organismo. Por vezes, a infecção pode estar localizada em apenas um órgão, como por exemplo, o pulmão. Contudo, provoca em todo o organismo uma resposta, a inflamação, numa tentativa de combater o agente da infecção. Essa inflamação pode vir a comprometer o funcionamento de vários órgãos do paciente (INSTITUTO LATINO AMERICANO DE SEPSE, 2018b)

Segundo o Instituto Latino Americano de Sepse (ILAS, 2019) a Sepse pode ser definida como a presença de disfunção orgânica que coloca a vida em risco em decorrência da resposta desregulada do organismo a presença de uma infecção, seja ela determinada por bactérias, vírus, fungos ou protozoários. Manifestando-se em diferentes estágios clínicos de um mesmo processo fisiopatológico, como traz o quadro 1 a seguir. Ela é, para o profissional de saúde de praticamente todas as especialidades, uma provocação, pela necessidade de pronto reconhecimento e tratamento precoce. 
Quadro 1. Definições de SIRS, Sepse, Sepse Grave e Choque Séptico.

\begin{tabular}{|c|c|}
\hline $\begin{array}{l}\text { Síndrome de Resposta } \\
\text { Inflamatória Sistêmica - } \\
\text { SIRS }\end{array}$ & $\begin{array}{l}\text { A síndrome da resposta inflamatória sistêmica é } \\
\text { definida pela presença de no mínimo dois dos } \\
\text { sinais abaixo: } \\
\text { - temperatura central }>38,3^{\circ} \mathrm{C} \text { ou }<36^{\circ} \mathrm{C} \mathrm{OU} \\
\text { equivalente em termos de temperatura axilar; } \\
\text { - frequência cardíaca }>90 \mathrm{bpm} \text {; } \\
\text { - frequência respiratória }>20 \mathrm{rpm} \text {, ou PaCO2 < } \\
32 \text { mmHg } \\
\text { - leucócitos totais }>12.000 / \mathrm{mm}^{3} \text {; ou }<4.000 / \mathrm{mm}^{3} \\
\text { ou presença de }>10 \% \text { de formas jovens }(\text { desvio } \\
\text { à esquerda). }\end{array}$ \\
\hline Sepse & $\begin{array}{l}\text { Infecção suspeita ou confirmada, sem disfunção } \\
\text { orgânica, de forma independente da presença de } \\
\text { sinais de SRIS. }\end{array}$ \\
\hline Sepse Grave & $\begin{array}{l}\text { Infecção suspeita ou confirmada associada a } \\
\text { disfunção orgânica, de forma independente da } \\
\text { presença de sinais de SRIS. }\end{array}$ \\
\hline Choque Séptico & $\begin{array}{l}\text { Sepse que evoluiu com hipotensão não corrigida } \\
\text { com reposição volêmica (PAM } \leq 65 \mathrm{mmHg} \text { ), de } \\
\text { forma independente de alterações de lactato }\end{array}$ \\
\hline
\end{tabular}

Fonte: Instituto Latino Americano para estudos da Sepse - ILAS. Roteiro de implementação de protocolo assistencial gerenciado. Campanha de Sobrevivência à Sepse. São Paulo: ILAS;2019.

É uma síndrome muito prevalente, com elevada taxa de morbimortalidade bem como elevação dos custos para seu tratamento. Seu reconhecimento precoce e tratamento adequado são fatores primordiais para a mudança deste cenário. $A$ construção de protocolos clínicos é uma ferramenta útil para o controle desta problemática, auxiliando as instituições na padronização do atendimento, diminuindo desfechos negativos e proporcionando melhor direcionamento do cuidado (ILAS, 2019). 
A sepse torna-se um importante problema socioeconômico para a saúde pública no mundo, por ser uma das principais causa de óbito nas Unidades de Terapia Intensiva (UTI). Acomete ao ano, milhões de pessoas, com altas taxas de mortalidade, igualando-se aos casos de infarto agudo do miocárdio, acidente vascular cerebral e poli trauma (COREN, 2017). Estudos abordam a sepse como uma das principais causas de morte em unidades de terapia intensiva (UTIs) não cardiológicas, entretanto, as taxas de mortalidade podem diferir de acordo com as condições socioeconômicas de cada país (ALMEIDA, 2013).

Segundo Viana, Machado e Souza (2017) uma avaliação feita no Brasil estimou os custos associados ao tratamento da sepse em US $\$ 9,6$ mil por paciente. Esses custos elevados se dão devido à necessidade de se utilizar medicamentos caros, equipamentos sofisticados e por exigir seguimento minucioso do paciente por parte da equipe médica e de enfermagem. Para os profissionais de saúde, a redução do número de pessoas que morrem por sepse é vista como um desafio diário. Sabe-se que para isso o melhor a se fazer é prevení-la, no entanto, se a mesma já estiver presente no paciente, é de suma importância que o diagnóstico e o tratamento sejam feitos o mais rápido possível.

É importante observar que o problema brasileiro de saúde pública da sepse não se restringe apenas à morbimortalidade elevadas, mas também às complicações que os sobreviventes da sepse podem desenvolver no pós alta, como problemas de saúde nas áreas física, cognitiva ou mental, decorrentes do processo saúde doença, das medidas terapêuticas recebidas bem como o prolongado tempo de internação (ARAUJO, 2016).

A sepse é diagnosticada, frequentemente, de forma tardia, já que os sinais e os sintomas atualmente utilizados para o diagnóstico, como alterações na contagem de leucócitos, febre, taquicardia e taquipneia, não são específicos da sepse. Concomitantemente, considera-se o conhecimento insuficiente dos profissionais da saúde, podendo ter inúmeras causas como o déficit na formação e a falta de definições oficiais precisas e processos adequados para ajudar na identificação e tornar o planejamento de cuidados mais rápido e efetivo (ZONTA et al., 2018).

Para que a sepse seja identificada e tratada o mais precocemente possível e seu desfecho seja positivo, diversos guidelines preconizam as melhores práticas baseadas em evidências, dentre eles o guideline do Surviving Sepsis Campaign que objetivou criar um esforço de colaboração internacional para melhorar o tratamento 
da sepse e reduzir a alta taxa de mortalidade associada a esta condição (DELLINGER, 2015). Dessa forma é importante estar atento e avaliar diariamente os usuários em estado grave e potencialmente infectados para sepse grave, a fim de promover a identificação precoce da doença e implementação precoce da terapia.

Portanto, a atuação de toda a equipe assistencial se faz imprescindível no atendimento ao usuário com diagnostico suspeito ou confirmado de sepse grave ou choque séptico, uma vez que é a equipe que está mais próxima do usuário durante todo o momento do cuidado, além de ser aquela que quando devidamente treinada, é capaz de identificar precocemente os sinais e sintomas de alerta para sepse, assim como implementar as intervenções específicas preconizadas pelos guidelines e protocolos institucionais (COFEN, 2017).

Para que a identificação seja precoce e o tratamento adequado, é fundamental a aplicação efetiva dos protocolos de sepse preconizados pelo ILAS e a capacitação efetiva dos profissionais de saúde. Revela-se aqui o papel valioso do enfermeiro como membro da equipe multiprofissional, pois ele identifica os sinais e os sintomas de sepse, assim como os riscos para seu desenvolvimento (SILVA, 2006).

Nesse sentido, observou-se a necessidade da implementação institucional da ferramenta facilitadora de Protocolos Assistenciais Multiprofissionais. Estes protocolos são padronizações de formas estruturadas de suporte do manejo clínico e que abrangem uma definição de objetivos terapêuticos e uma sequência temporal de cuidados e estratégias diagnósticas e terapêuticas definidas. São elaborados e implementados pela equipe multiprofissional e geralmente gerenciados por um profissional da área da saúde (SANTOS, 2015).

Nesta perspectiva, a construção de um instrumento prático e sistematizado, embasado nas diretrizes internacionais, compatíveis com a realidade local, esperase contribuir para a atuação da equipe assistencial no diagnóstico e tratamento precoces da sepse, na busca da diminuição da mortalidade dos usuários no serviço, o tempo de hospitalização e consequentemente os custos, além de identificar precocemente sinais de deterioração clínica, prezando impreterivelmente pela segurança em todos os aspectos do cuidado em saúde.

Para que esse movimento ocorra, é necessário que gestores e líderes, colaboradores e os próprios usuários entendam o significado e a importância da implementação dessas estratégias institucionais e é imprescindível que todos 
estejam envolvidos nesta causa, visando um único objetivo: tornar o cuidado em saúde cada vez mais seguro.

\section{OBJETIVOS}

\subsection{Geral}

Propor a construção de um protocolo para manejo da assistência da equipe multiprofissional ao paciente séptico em Unidades de emergência.

\subsection{Específicos}

a) Desenvolver um protocolo assistencial, com os passos a serem seguidos pela equipe assistencial ao paciente com sepse;

b) Propor a criação de um modelo de ficha de triagem e um fluxograma de atendimento ao paciente com sinais de sepse;

c) Desenvolver ferramentas para o reconhecimento e abordagem precoce do profissional ao paciente. 


\section{METODOLOGIA}

\subsection{Delineamento}

Trata-se de um estudo de desenvolvimento metodológico e descritivo, para a elaboração de um protocolo assistencial baseado nas melhores evidências clínicas. No estudo metodológico, o pesquisador tem como meta a elaboração de um instrumento confiável, preciso e utilizável que possa ser empregado por outros pesquisadores e outras pessoas. Esse estudo cabe a qualquer disciplina científica, lidando com fenômenos complexos como o comportamento ou a saúde dos indivíduos, tal qual ocorre na pesquisa de enfermagem (POLIT; BECK, 2011).

Neste processo, busca-se o entendimento aprofundado da problemática e das pessoas envolvidas na construção da intervenção, tendo um planejamento criterioso e coordenado, além do desenvolvimento teórico da intervenção para nortear a investigação. (POLIT; BECK; HUNGLER, 2011).

As diretrizes internacionais recomendam que todas as instituições de saúde lancem mão de estratégias para a identificação de sepse. Um estudo desenvolvido pelo ILAS, no Brasil, evidenciou considerável redução da letalidade em estabelecimentos de saúde que implementaram protocolos de detecção desse processo patológico. Além disso, pode ser observada uma redução de gastos para com os pacientes com sepse (VIANA; MACHADO; SOUZA, 2017).

Entretanto, nem todas as instituições de saúde utilizam protocolos para identificação de sepse. Em sua pesquisa, Guarrido (2017) observaram que em aproximadamente metade dos estabelecimentos de saúde pesquisados, não são utilizados protocolos ou impressos específicos para detecção desse processo patológico. Tal fato prejudica a atuação do enfermeiro e demais profissionais de saúde, uma vez que, muitas vezes, estes não são capacitados para desenvolver essa tarefa, por falta de ações de educação permanente propostas pelas próprias instituições.

Vale ressaltar que, para que os protocolos de identificação de manejo sejam eficazes, é importante que abordem pontos como as principais disfunções orgânicas, peculiaridades do tratamento e aspectos sobre letalidade (ILAS, 2017). Destarte, é relevante que além desses protocolos, as instituições de saúde promovam capacitações sobre o assunto, para que os profissionais possam agir com destreza quando se depararem com situações de sepse. 


\subsection{Local e período da pesquisa}

O presente estudo foi desenvolvido no hospital Santa Casa de Misericórdia de Sobral, situado na região norte do estado do Ceara. É uma instituição filantrópica de referência regional e estadual para atendimento a população de 55 municípios da Macrorregião de Sobral/CE com uma população de aproximadamente 1.720 .000 de habitantes que procuram atendimento de Alta Complexidade em Traumatoortopedia, Obstetrícia, Neurocirurgia, Oncologia, Cardiologia e Terapia Renal Substitutiva, sendo certificado como Hospital de Ensino pelos Ministérios da Educação e da Saúde desde 2007. Esta instituição é caracterizada como hospital de ensino, referência em residências médicas, cirúrgicas e multiprofissionais. A SCMS atende a população de 55 municípios da Macrorregião de Sobral/CE com uma população de aproximadamente 1.720 .000 de habitantes (SANTA CASA DE MISERICÓRDIA DE SOBRAL)

\subsection{Definição do problema}

O problema de pesquisa foi definido através da pergunta: quais os critérios e riscos para identificação do paciente séptico em um unidade de terapia intensiva? Este questionamento guiou a revisão dos estudos que serão incluídos no protocolo.

\subsection{Métodos e procedimentos}

O Protocolo a proposto contem passos a serem seguidos pela equipe de saúde para a assistência durante o período de sepse, em cada fase, de acordo com as condições clínicas do paciente. Com base na busca da literatura nacional e internacional em consonância com o Instituto Latino-Americano de Sepse e seus manuais de manejo ao paciente.

Além do Protocolo, foi proposto um modelo de fluxograma para a abertura do protocolo assistencial de sepse na UTI bem como uma ficha de triagem para estes pacientes, com vistas a facilitar a conduta clínica do profissional, descrevendo as etapas de forma direta e autoexplicativa, ambos com base em modelos criados pelo ILAS (2019).

\subsection{Aspectos éticos e legais}

O estudo atendeu a Resolução n. 466/12, do Conselho Nacional de Saúde, que estabelece critérios éticos para pesquisa com seres humanos. $O$ estudo foi 
realizado mediante a aprovação pelo Comitê de Ética em Pesquisa (CEP) do Hospital Santa Casa de Misericórdia de Sobral.

$\mathrm{O}$ estudo respeitou as exigências formais contidas nas normas nacionais e internacionais regulamentadoras de pesquisas envolvendo seres humanos, sendo encaminhada ao comitê de ética em pesquisa do hospital. Todos os itens dispostos na Resolução 466, de 12 de dezembro de 2012, do Conselho Nacional de Saúde, que regulamenta a pesquisa com seres humanos, serão obedecidos, especialmente quanto à explicação dos objetivos, finalidade e riscos do estudo, além da garantia do anonimato dos mesmos e do direito de se retirarem da investigação a qualquer momento, sem que isso acarrete algum prejuízo.

Após a qualificação do projeto o mesmo foi enviado a Comissão Cientifica e Ética da Santa Casa de Sobral localizado na Rua Antônio Crisóstomo de Melo, $n^{\circ}$ 919, Bairro Centro, Sobral- CE, tendo parecer favorável.

\section{RESULTADOS E DISCUSSÕES}

\subsection{Etapas para a construção do protocolo assistencial}

A primeira etapa deste estudo foi a busca e Análise de informações. $O$ protocolo foi desenvolvido a partir de um levantamento em literatura nacional e internacional. O Protocolo proposto contem passos a serem seguidos pela equipe de saúde para identificação e manejo do paciente com sepse, de acordo com as condições clínicas do paciente.

Para a elaboração de um protocolo assistencial de boa qualidade é necessário rigor metodológico em todas as suas etapas, optou-se então por iniciar a construção deste instrumento pela revisão sistemática da literatura que é o método mais apropriado para a elaboração de um protocolo (ECCLES et al., 2012; INSTITUTE OF MEDICINE, 2011). O fluxograma abaixo demonstra os principais passos seguidos para esta revisão (Figura1).

Segundo Nascimento e Draganov (2015) este tipo de pesquisa é tida como uma estratégia que faz uso de modo sistemático dos conhecimentos existentes para a criação de uma nova intervenção ou que possibilite a melhora significativa de uma intervenção já existente, ou mesmo, desenvolva ou aprimore um instrumento, um dispositivo ou um método de mediação. 
Figura 1: Fluxograma para revisão sistemática

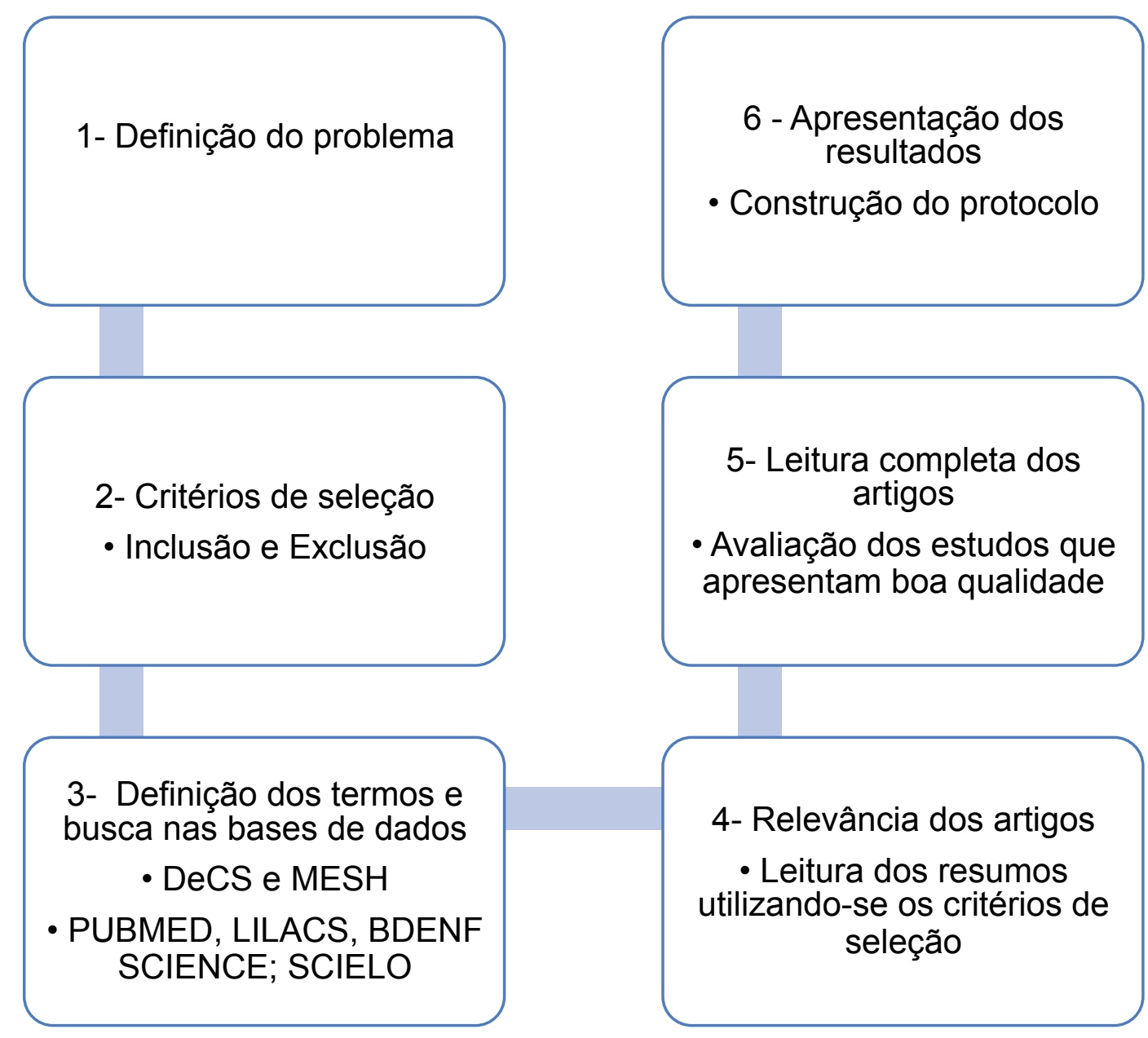

Fonte: elaborado pelo autor (2020)

\subsection{Critérios de seleção}

Para a seleção dos artigos, foram utilizados os seguintes critérios:

Critérios de inclusão dos artigos:

a) artigos que retratem o tema do estudo;

b) estejam disponíveis gratuitamente e na íntegra nas bases de dados pesquisadas;

c) terem sido publicados a partir do ano de 2015;

d) teses e dissertações de revisão sistemática ou metanálise.

Critérios de exclusão dos artigos:

a) resumos e resumos expandidos; 
b) artigos publicados em anais de congressos e que não estejam disponibilizados na íntegra;

c) livros.

Os que não atenderem os critérios estabelecidos serão excluídos.

\subsection{A Construção do protocolo sepse}

\section{$2^{\circ}$ etapa - construção do protocolo assistencial}

Para realizar construção do protocolo de sepse de forma institucional, o protocolo de sepse (APÊNDICE A) foi elaborado a partir da necessidade do seu gerenciamento de forma institucional. Para a identificação do paciente com suspeita de sepse os seguintes passos devem ser cumpridos como: registrar o diagnóstico no prontuário ou na folha específica de triagem do protocolo institucional; todas as medidas devem ser tomadas a partir do momento da formulação da hipótese de sepse.

Todos os pacientes com protocolos de sepse abertos devem ter seu atendimento priorizado, com o objetivo de otimizar a coleta de exames, o início de antibioticoterapia e a ressuscitação hemodinâmica; realizar anamnese e exame físico dirigidos, com atenção especial aos sinais clínicos de disfunção orgânica; pacientes com disfunção orgânica grave e ou choque devem ser alocados em leitos de terapia intensiva assim que possível, a fim de garantir o suporte clínico necessário.

Caso não seja possível a alocação em leito de terapia intensiva, deve-se garantir o atendimento do paciente de maneira integral, independente do setor em que ele se encontra; e a ficha do protocolo de sepse deve acompanhar o paciente durante todo $o$ atendimento de tratamento nas primeiras horas, a fim de facilitar a comunicação nos pontos de transição entre as equipes de diferentes turnos ou setores e resolver pendências existentes para o atendimento (INSTITUTO LATINO AMERICANO DE SEPSE, 2018b).

\subsection{Construção da ficha de triagem e fluxograma para pacientes com suspeita de sepse}

Nesta terceira etapa, o protocolo deve ser iniciado para os pacientes com suspeita de sepse e choque séptico seguindo o modelo de fluxograma de atendimento proposto (APÊNDICE B) e ficha para triagem (APÊNDICE C). A 
instituição decidirá, de acordo com a sua disponibilidade de recursos humanos e capacidade de triagem, que o protocolo de sepse será aberto na presença de SRIS (Síndrome da resposta inflamatória sistêmica) e de e suspeita de infecção (elevada sensibilidade, permitindo tratamento precoce e prevenindo disfunção orgânica), ou a partir de presença de disfunção orgânica em pacientes com suspeita de infecção grave, priorizando, nesse caso, o atendimento daqueles mais graves.

A presença de disfunção orgânica clínica também desencadeia a suspeita de sepse. A abertura de protocolos com base apenas na presença de disfunção orgânica promove o diagnóstico num estágio mais avançado da síndrome. Por outro lado, o acionamento apenas com base nos critérios de SRIS (Síndrome da resposta inflamatória sistêmica) torna a ferramenta mais sensível. De uma forma ou outra, a responsabilidade prioritária para essa detecção é da enfermagem, e esses profissionais devem ser especificamente e rotineiramente treinados para tal fim.

A enfermagem, ao identificar um paciente potencial, pode proceder à abertura do protocolo, com o preenchimento da ficha e o acionamento da equipe médica. A avaliação médica define se há ou não foco infeccioso suspeito como causador da SRIS (Síndrome da resposta inflamatória sistêmica), ou seja, o médico define se será dada continuidade ao atendimento com base no diagnóstico de possível sepse ou se deve ser afastado. A equipe médica pode optar por não dar seguimento ao protocolo em pacientes em cuidados de fim de vida ou com doenças infecciosas específicas, como dengue, leptospirose dentre outras.

A instituição deve ser capaz de identificar todos os pacientes com suspeita de sepse, de forma a permitir o diagnóstico precoce. Todos os setores do hospital devem utilizar instrumentos de triagem, para detecção precoce do paciente séptico.

\section{CONSIDERAÇÕES FINAIS}

Considerando a magnitude da sepse no cenário mundial, o estudo mostrou um amplo referencial teórico em que a prevenção tem sido apontada como o melhor caminho para minimizar os eventos adversos, com enfoque na utilização de estratégias de gestão clínica. Novas estratégias de prevenção estão presentes na literatura para a redução de sua incidência associadas à busca por atendimento de qualidade e aumento da qualidade de vida do paciente.

Este estudo possibilitou a criação de um protocolo de gerenciamento de sepse em um hospital de grande porte em Sobral, Ceará. Diante da suspeita de 
sepse em pacientes internados, gerenciar um protocolo que permite a identificação precoce e que assegura intervenções eficazes logo na primeira hora de assistência deve ser o objetivo de toda gestão clínica. Todo estudo tem limitações e este não é exceção. Assim, para realização desta pesquisa ao longo da construção do protocolo, foram levantadass possíveis dificuldades e limitações, merecendo destaque:

- Engajamento do corpo clínico;

- Estrutura física e funcional da unidade;

- Criação de fluxos de priorização ao paciente séptico;

- Equipe multidisciplinar com o olhar somente para o seu setor e não de forma institucional, dificultando a disseminação do protocolo;

- Processo novo com pouca adesão ao protocolo de sepse pelos médicos, enfermeiros e técnicos de enfermagem;

- Baixa adesão ao protocolo na unidade de terapia intensiva por toda euipe assistencial, uma vez que é um protocolo novo.

O estudo evidencia que o protocolo não é apenas mais um documento da assistencial, e sim, uma ferramenta importante para prestar melhor cuidado de toda equipe multiprofissional. Assim, o protocolo trará benefícios para a prática assistencial, agilidade para o atendimento e qualificação para a equipe.

Espera-se que com este protocolo implantado no hospital, possa contribuir para o melhor desempenho dos profissionais de saúde. Que os resultados deste estudo sejam discutidos entre os membros das equipes envolvidas e que delas surjam reflexões sobre a possibilidade de maximizar a adesão ao protocolo com o intuito de salvar vidas. Além disso, que estes resultados possam subsidiar novos estudos e pesquisas sobre o tema. 


\section{REFERÊNCIAS}

ALMEIDA, A. P. S. R. et al. Conhecimento do profissional enfermeiro a respeito da Sepse. Brazilian Journal of Surgery and Clinical Research, Rondônia, v.4, n.4, p. 5-10, set./nov. 2013. Disponível em: <http://biblioteca.cofen.gov.br/wpcontent/uploads/2015/07/Conhecimento-do-profissional-enfermeiro-a-respeito-daSEPSE.pdf>. Acesso em 06 dez de 2019.

ARAÚJO, B. Protocolo multidisciplinar institucional. Hospital Vera Cruz, 2016. Disponível em:

<http://www.hospitalveracruz.com.br/conteudo/arquivo/2017/mar/hospital-vera-cruzcampinas_1490193401_pmi083\%20protocolo\%20de\%20sepse\%20no\%20adulto.pdf >. Acesso em: 06 dez de 2020.

ARAÚJO, M. L. Identificação da sepse pela equipe de enfermagem em um serviço de emergência de um hospital geral. 2014. 25p. Trabalho de Conclusão de Curso (Especialização em Linhas de Cuidado em Enfermagem) - Universidade Federal de Santa Catarina-UFSC, Florianópolis, 2014.

BROUWERS, Melissa et al. AGREE Next Steps Consortium. AGREE II: Advancing guideline development, reporting and evaluation in healthcare. Can Med Assoc J., Ottawa, v. 182, p. E839-E842, 2010. doi: 10.1503/cmaj.090449.

CONSELHO FEDERAL DE ENFERMAGEM, 2017. Disponível em: $<$ http://www.cofen.gov.br/mais-de-cem-municipios-reduziram-para-30h-a-jornadados-profissionais-de-enfermagem_48298.html>. Acesso em: 06 dez de 2020.

Dellinger RP, Casserly B, Phillips GS, Schorr C, Townsend SR, Osborn TM, et al. Lactate measurements in sepsis-induced tissue hypoperfusion: results from the Surviving Sepsis Campaign database. Crit Care Med[Internet]. 2015;43(3):567- 73.: disponível: https://www.ncbi.nlm.nih.gov/pubmed/25479113. Acesso em 22 out 2020. 
DRAGANOV, B. P. NASCIMENTO, C. J. História da qualidade em segurança do paciente. Hist enferm: Rev. eletrônica, v. 6, n. 2, p. 299-309, 2015. Disponível em: . Acesso em: 20 de dez 2019.

ECCLES, Martin P. et al. Developing clinical practice guidelines: target audiences, identifying topics for guidelines, guideline group composition and functioning and conflicts of interest. Implementat Sci., London, v. 7, 8 p., 2012.

FUNK, D. J.; PARRILLO, J. E.; KUMAR, A. Sepsis and septic shock: a history. Critical Care Clinics, v. 25, n. 1, p. 83-101, 2009.

GUARRIDO, F. et al. Ações do enfermeiro na identificação precoce de alterações sistêmicas causadas pela sepse grave. ABCS Health Sciences, São Pulo, v. 42, n. 1, p. 15-20, 2017. Disponível em:

<https://www.portalnepas.org.br/abcshs/article/view/944/756>. http://dx.doi.org/10.7322/abcshs.v42i1.944. Acesso em: 02 out 2019.

INSTITUTE OF MEDICINE. IOM. Finding what works in health care. Standards for systematic reviews. Rep Brief. 2011. Disponível em:

<http://www.iom.edu/Reports/2011/Finding-What-Works-in-Health-Care standardsfor-Systematic-Reviews/Report-Brief.aspx>. Acesso em: 28 de nov 2020.

INSTITUTO LATINO-AMERICANO DE SEPSE. Implementação de protocolo gerenciado de sepse. Protocolo clínico. Jun. 2019, 14p. Disponível em: < http://www.ilas.org.br/assets/arquivos/ferramentas/protocolo-de-tratamento.pdf>. Acesso em: 06 de nov 2020.

INSTITUTO LATINO-AMERICANO DE SEPSE. Roteiro de implementação de protocolo assistencial gerenciado. $4^{a}$ ed. São Paulo: ILAS, 2018. Disponível em: <http://www.ilas.org.br/assets/arquivos/ferramentas/roteiro-de-implementacao.pdf>. Acesso em: 06 nov de 2019. 
POLIT D. F.; BECK C. T. Fundamentos de Pesquisa em enfermagem: avaliação de evidências para as práticas da enfermagem. 7. ed. Porto Alegre, Rio Grande do Sul, 2011.

SANTOS, V. C.; et al. Perfil epidemiológico da sepse em um hospital de urgência. Revista prevenção de infecção e saúde, v. 1, n. 1, fev. 2015. Disponível em: < http://www.ojs.ufpi.br/index.php/nupcis/article/view/3154/pdf>. Acesso em: 10 jan 2021.

VIANA, R. A. P. P.; MACHADO; F. R.; SOUZA; J. L. A. Sepse, um problema de saúde pública: a atuação da enfermagem na rápida identificação e tratamento da doença. Conselho Regional de Enfermagem-SP. $2^{\mathrm{a}}$ ed. São Paulo, 2017, 65p. Disponível em: <http://www.ilas.org.br/assets/arquivos/ferramentas/livro-sepse-umproblema-de-saude-publica-coren-ilas.pdf>. Acesso em: 11 out 2020.

HENKIN, C. S. et al. Sepse: uma visão atual. Scientia Medica, Porto Alegre, v. 19, n. 3, p. 135-145, jul./set. 2009 
ANEXOS 


\section{ANEXO A - Protocolo de atendimento - Pacotes de 1 hora e de 6 horas}

\section{INSTITUTO LATINO AMERICANO DE SEPSE}

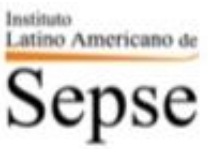

comunicação nos pontos de transiçăo entre as equipes de diferentes turnos ou setores e resolver pendências existentes para o atendimento.

\section{Tratamento}

\subsection{Pacote de 1 hora}

Para todos os pacientes em que a equipe médica optou por dar seguimento ao protocolo, o pacote de 1 hora deve ser executado. 0 mesmo é composto dos seguintes itens:

1. Coleta de exames laboratoriais para a pesquisa de disfunçōes orgânicas: gasometria e lactato arterial, hemograma completo, creatinina, bilirrubina e coagulograma.

2. Coleta de lactato arterial o mais rapidamente possível mas dentro da primeira hora, que deve ser imediatamente encaminhado ao laboratório, afim de se evitar resultado falsos positivos. O objetivo é ter resultado deste exame em 30 minutos.

3. Coleta de duas hemoculturas de sítios distintos em até uma hora, conforme rotina especifica do hospital, e culturas de todos os outros sítios pertinentes (aspirado traqueal, liquor, urocultura) antes da administração do antimicrobiano. Caso năo seja possivel a coleta destes exames antes da primeira dose, a administraçăo de antimicrobianos não deverá ser postergada;

4. Prescrição e administração de antimicrobianos de amplo espectro para a situação clínica, por via endovenosa, visando o foco suspeito, dentro da primeira hora da identificação da sepse. A utilizaçăo de antimicrobianos deve seguir a orientação do serviço de controle de infecção hospitalar da instituição, que deverá definir com o corpo clínico local as recomendaçōes para o 


\section{INSTITUTO LATINO AMERICANO DE SEPSE}

tratamento empirico conforme o foco de infecção identificado e a característica da infecção, comunitária ou associada a assistência a saúde.

5. Princípios de farmacocinética e farmacodinâmica devem ser seguidos por todas as instituições. Todas as recomendações visando otimização da terapia antimicrobiana devem ser feitas com auxilio do farmacêutico e da enfermagem e estarem amplamente disponiveis para todos os profissionais. As principais recomendações estão listadas abaixo.

- Utilizar dose máxima para o foco suspeito ou confirmado, com dose de ataque nos casos pertinentes, sem ajustes para a função renal ou hepática. As doses devem ser plenas visando otimizaçăo da redução da carga bacteriana ou fúngica. Embora seja discutível, pode-se manter doses sem ajuste para função renal pelas primeiras 24 horas. Isso é de suma importância para os antimicrobianos hidrofílicos dado ao aumento do volume de distribuição dos mesmos em decorrência da resuscitação volêmica.

- Atentar para a dilução adequada de forma a evitar incompatibilidade e concentração excessiva. Utilizar a infusāo estendida de antibióticos betalactâmicos como piperacilina-tazobactam e meropenem, com exceçăo da primeira dose, que deve ser administrada, em bolus, o mais rápido possível.

- Utilizar terapia combinada, com duas ou três drogas, quando existir suspeita de infecção por agentes multidrogas resistentes. Considerar o uso de diferentes classes de antibióticos, para um mesmo agente, em pacientes com choque séptico.

- Restringir o espectro antimicrobiano quando o patógeno for identificado e a sensibilidade conhecida; terapia combinada pode ser de-escalonada conforme evidência de resposta clínica ou resolução da infecção.

6. Para pacientes hipotensos (PAS $<90 \mathrm{mmHg}, \mathrm{PAM}<65 \mathrm{mmHg}$ ou, eventualmente, redução da PAS em $40 \mathrm{mmHg}$ da pressão habitual) ou com sinais de hipoperfusão, entre eles niveis de lactato acima de duas vezes o valor de referência institucional (hiperlactatemia inicial), deve ser iniciada ressuscitação volêmica com infusão imediata de $30 \mathrm{~mL} / \mathrm{kg}$ de cristalóides 


\section{INSTITUTO LATINO AMERICANO DE SEPSE}

dentro da $1^{\mathrm{a}}$ hora do diagnóstico da detecçaō dos sinais de hipoperfusão. Embora classicamente não sejam considerados com parte do pacote de resuscitação, sinais de hipoperfusão podem incluir oligúria, presença de livedo, tempo de enchimento capilar lentificado e alteração do nível de consciência. Coloides proteicos, albumina ou soro albuminado, podem fazer parte dessa reposição inicial. $\mathrm{O}$ uso de amidos está contraindicado, pois está associado a aumento da incidência de disfunção renal. Esse volume deve ser infundido o mais rápido possivel, considerando-se as condiçōes clínicas de cada paciente. Pacientes cardiopatas podem necessitar reduçăo na velocidade de infusão, conforme a presença ou năo de disfunção diastólica ou sistólica. Nos casos em que foi optado por não realizar reposição volêmica, parcial ou integralmente, após avaliação de fluido responsividade, esta decisão deve estar adequadamente registrada no prontuário. Nesses pacientes, o uso de vasopressores para garantir pressăo de perfusăo adequada necessita ser avaliado.

7. Uso de vasopressores para pacientes que permaneçam com pressão arterial média (PAM) abaixo de 65 (após a infusăo de volume inicial), sendo a noradrenalina a droga de primeira escolha. Não se deve tolerar pressões abaixo de $65 \mathrm{mmHg}$ por periodos superiores a 30-40 minutos. Por isso, o vasopressor deve ser iniciado dentro da primeira hora nos pacientes em que ele está indicado. Em casos de hipotensão ameaçadora a vida, pode-se iniciar o vasopressor mesmo antes ou durante a reposição volêmica. É fundamental garantir pressão de perfusão enquanto se continua a reposição volêmica. Assim, o vasopressor pode ser iniciado em veia periférica, enquanto se providencia o acesso venoso central.

$\mathrm{O}$ uso de outros vasopressores pode ser necessário. Dentre os disponiveis, a recomendação é o uso de vasopressina, com intuito de desmame de noradrenalina ou como estratégia poupadora de catecolaminas, ou a adrenalina, preferivel em pacientes que se apresentem com débito cardiaco reduzido.

A dobutamina pode ser utilizada quando exista evidência de baixo cardiaco ou sinais clínicos de hipoperfusăo tecidual, como livedo, oligúria, tempo de 


\section{INSTITUTO LATINO AMERICANO DE SEPSE}

enchimento capilar lentificado, baixa saturação venosa central ou lactato aumentado.

8. Nos pacientes com lactato alterado acima de duas vezes o valor de referência, a meta terapêutica é o clareamento do mesmo. Assim, como um complemento ao pacote de 1 hora, dentro de 2 a 4 horas após o início da ressuscitação volêmica, novas dosagens devem ser solicitadas. Nem sempre se obtem a normalização do lactato, haja vista existirem outras causas para a hiperlactatemia que não a hipoperfusão tecidual. $\mathrm{A}$ busca pela normalização deve ser feita cuidadosamente, sob risco de intervençōes terapêuticas desnecessárias, e potencialmente deletérias. A hiperlactatemia residual isolada, sem outros sinais clínicos de hipoperfusão ou má evolução, não necessariamente precisa ser tratada.

\subsection{Reavaliação das 6 horas}

A reavaliação das 6 horas deve ser feita em pacientes que se apresentem com choque séptico, hiperlactatemia ou sinais clínicos de hipoperfusão tecidual.

A continuidade do cuidado é importante, por isso entende-se que durante as seis primeiras horas o paciente deve ser reavaliado periodicamente. Para isso é importante o registro da reavaliação do status volêmico e da perfusão tecidual.

1. Reavaliação da continuidade da ressuscitação volêmica, por meio de marcadores do estado volêmico ou de parâmetros perfusionais. As seguintes formas de reavaliação poderão ser consideradas:

- Mensuração de pressão venosa central

- Variação de pressão de pulso

- Variação de distensibilidade de veia cava

- Elevação passiva de membros inferiores

- Qualquer outra forma de avaliação de responsividade a fluidos (melhora da pressão arterial após infusão de fluidos, por exemplo)

- Mensuração de saturação venosa central 
INSTITUTO LATINO AMERICANO DE SEPSE

- Tempo de enchimento capilar

- Presença de livedo

- Sinais indiretos (por exemplo, melhora do nivel de consciência ou presença de diurese)

2. Pacientes com sinais de hipoperfusão e com níveis de hemoglobina abaixo de $7 \mathrm{mg} / \mathrm{dL}$ devem receber transfusão o mais rapidamente possível.

3. Idealmente, os pacientes com choque séptico devem ser monitorados com pressão arterial invasiva, enquanto estiverem em uso de vasopressor. A aferição por manguito não é fidedigna nessa situação, mas pode ser utilizada nos locais onde a monitorização invasiva não está disponivel.

4. Pacientes sépticos podem se apresentar hipertensos, principalmente se já portadores de hipertensão arterial sistêmica. Nesses casos, a redução da póscarga pode ser necessária para o restabelecimento da adequada oferta de oxigênio. Não se deve usar medicações de efeito prolongado, pois esses pacientes podem rapidamente evoluir com hipotensão. Assim, vasodilatadores endovenosos, como nitroglicerina ou nitroprussiatos são as drogas de escolha 


\section{APÊNDICES}




\section{APÊNDICE A - Protocolo de sepse PROTOCOLO CLÍNICO MULTIDISCIPLINAR}

\begin{tabular}{|l|}
\hline 1) Atividade \\
Protocolo de Sepse \\
\hline 2) Responsável (Cargo de quem realiza a atividade) \\
Equipe Multidisciplinar \\
\hline
\end{tabular}

\section{Siglas e definições}

UTI - Unidade de Terapia Intensiva

UNI - Unidade de Internação

UNE - Unidade de Emergência

SIRS - Síndrome de resposta inflamatória sistêmica

SVO2 - Saturação venosa de oxigênio

$\mathrm{pH}$ - Potencial Hidrogeniônico

PAM - Pressão arterial média

PVC - Pressão venosa central

PAS - Pressão arterial sistólica

PA - Pressão arterial

O2 - Oxigênio

SSC - Surviving Sepsis Campaing/Campanha de Sobrevivência a Sepse ILAS Instituto Latino Americano de Sepse

VM - Ventilação mecânica

\section{Abrangência}

Este protocolo aplica-se as unidades de terapia intensiva - UTI adulta da Santa Casa de Misericórdia de Sobral.

\section{Diretrizes}

\section{1 - Introdução}

Sepse é definida pela presença de disfunção ameaçadora à vida em decorrência da presença de resposta desregulada à infecção.

A síndrome de resposta inflamatória sistêmica (SRIS), embora não utilizada para a 
definição de sepse, continua sendo importante para a triagem de pacientes com suspeita de sepse. Da mesma forma que a SSC (Surviving Sepsis Campaing/Campanha de Sobrevivência a Sepse), o ILAS (Instituto Latino Americano de Sepse) não mudou os critérios usados para definir disfunção orgânica, mantendo a hiperlactatemia como um deles. O critério para definição de choque séptico também não foi alterado. Segundo a SSC, choque séptico é definido pela presença de hipotensão não responsiva à utilização de fluídos, independente dos valores de lactato.

A SSC não adotou o novo conceito de choque, que exige a presença concomitante de lactato acima do valor de referência, mesmo após reposição volêmica inicial. Considerando a alta prevalência, elevada morbidade, mortalidade e alto custo relacionados à Sepse, o HFR definiu pela implementação e gerenciamento deste protocolo.

\section{2 - Objetivos}

- Estabelecer critérios para o reconhecimento precoce dos sinais de Sepse;

- Padronizar o atendimento aos pacientes com suspeita ou confirmação de Sepse, visando o melhor atendimento, tratamento e desfecho clínico.

\section{3 - Possíveis focos infecciosos}

- Pulmonar

- Abdominal

- Cardíaco

- Ósseo articular

- Sistema nervoso

- Urinário

- Ferida operatória

- Lesões de pele profundas

- Corrente sanguínea associada a cateter.

- Outros

\section{4- Critérios para SIRS}

- Hipertermia $>37,8^{\circ} \mathrm{C}$ ou hipotermia $<35^{\circ} \mathrm{C}$

- Taquicardia > 90 bpm

- Taquipneia $>20$ ipm ou PaCO2 $<32 \mathrm{mmHg}$ ou necessidade de ventilação mecânica; 


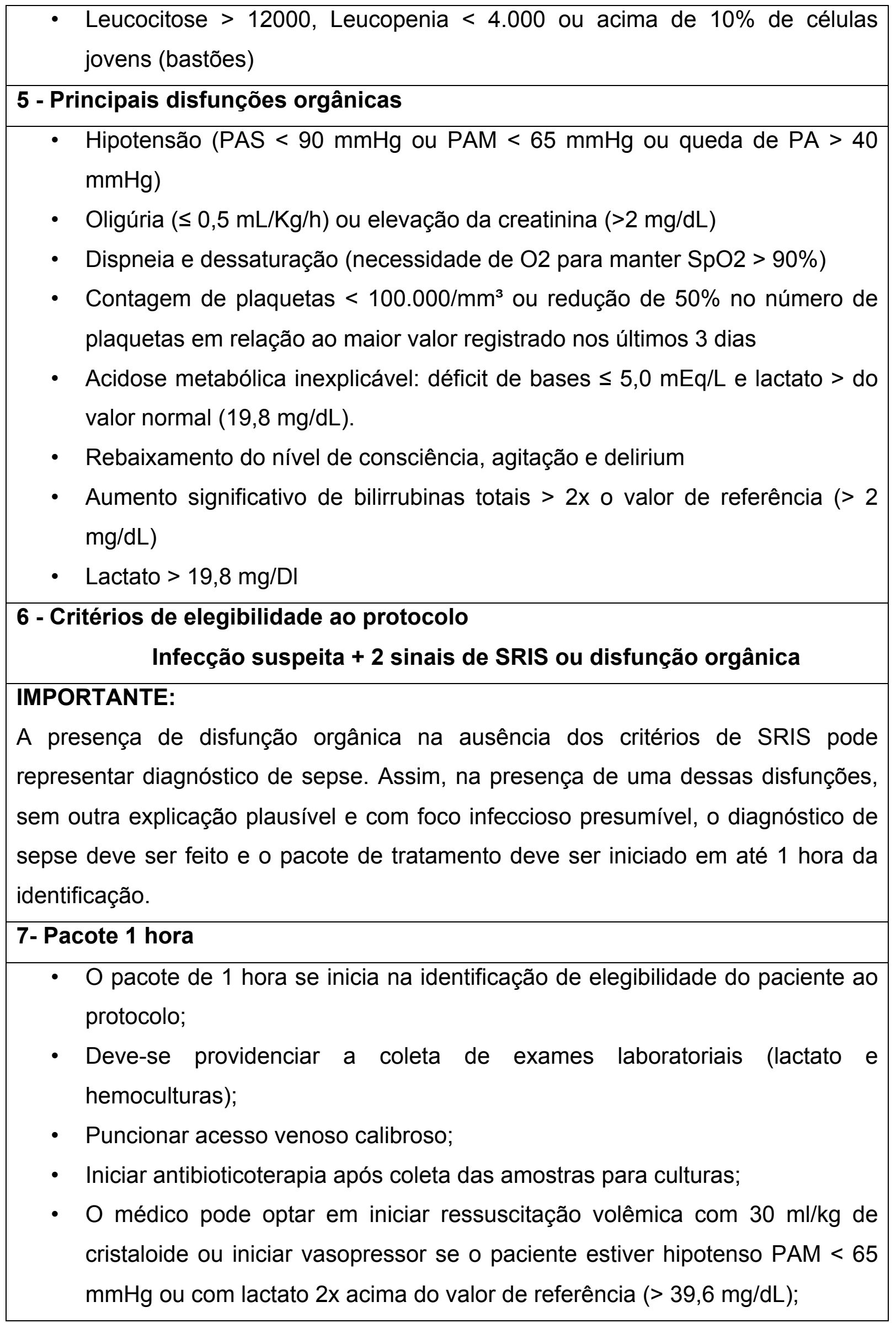


- Coletar $2^{\circ}$ amostra de lactato entre 2-4 horas para pacientes com hiperlactatemia, ou seja, quando o resultado do lactato for $2 x$ acima do valor de referência (> 39,6 mg/dL);

\section{8 - Competências da Equipe Multidisciplinar}

\section{TÉCNICO DE ENFERMAGEM}

- Aferir sinais vitais conforme prescrição de enfermagem/médico;

- Comunicar imediatamente ao enfermeiro caso o paciente apresente sinais sugestivos de sepse.

\section{ENFERMEIRO}

- Abrir o protocolo de sepse, acionar a equipe médica através do código amarelo, com comunicação efetiva, relatando ao médico que o paciente apresenta sinais sugestivos de sepse;

- Preencher o check-list de gerenciamento do protocolo de sepse;

- Acionar o laboratório, após solicitação dos exames pela equipe médica, no telefone 99215- 8683 ou 7394;

- Identificar o paciente como paciente crítico, mantendo monitoramento do paciente; - Garantir acesso venoso calibroso;

- Garantir a administração do antibiótico prescrito pelo médico em até 1 hora da identificação dos sinais de sepse;

- Registrar em prontuário.

\section{MÉDICO}

- Definir se dará continuidade ao protocolo de sepse ou encerrá-lo para seguir com tratamento individualizado fora do protocolo;

- Solicitar exames laboratoriais pela prescrição padrão: SEPSE (CTI/UNI ou pronto atendimento);

- Prescrever antibiótico e reposição volêmica se necessário e manter acompanhamento do paciente;

- Solicitar transferência do paciente para o CTI, quando o mesmo for elegível de acordo com os critérios de gravidade: choque séptico, hipotensão, hiperlactatemia, VM, etc.

- Realizar o registro em prontuário e comunicar a equipe de enfermagem.

\section{LABORATÓRIO}

- Coletar os exames laboratoriais em até 20 minutos após a abertura do 


\begin{tabular}{|l|}
\hline protocolo de sepse; \\
- Encaminhar as amostras de lactato e hemocultura imediatamente para \\
análise, o objetivo é ter o resultado do lactato em até 30 minutos da abertura \\
do protocolo de sepse. \\
- Encaminhar o resultado do lactato para o enfermeiro pessoalmente e via \\
sistema informatizado. \\
GESTÃO DE LEITOS \\
Disponibilizar leito em CTI, se necessário, em até 1 hora. \\
SERVIÇO DE CONTROLE DE INFECÇÃO HOSPITALAR \\
Manter atualizado e disponibilizar o quadro de antibioticoterapia empírica, \\
de acordo com o perfil epidemiológico e microbiológico.
\end{tabular}

\section{Registros}

Prontuário do paciente - Sistema MV PEP

Registros próprios do Serviço de Controle de Infecção Hospitalar Análise de indicadores próprios do setor

\section{Referências bibliográficas}

Instituto Latino Americano de Sepse, acesso em:

http://www.ilas.org.br/assets/arquivos/ferramentas/protocolo-de-tratamento.pdf

Levy, M.M., Evans, L.E. \& Rhodes, A. Intensive Care Med (2018), acesso em:

https://doi.org/10.1007/s00134-018-5085-0

PIMENTA, C. A. M. et al. Guia para construção de protocolo assistencial de enfermagem. São Paulo: COREN-SP, 2017. 


\section{APÊNDICE B - FLUXOGRAMA DE ATENDIMENTO PARA PACIENTES COM}

\section{SUSPEITA DE SEPSE}

\section{SIRIS}

FC $>90$

FR $>20$

$\mathrm{T}>37.8^{\circ} \mathrm{OU} \mathrm{T}<35,0^{\circ} \mathrm{C}$

LEUCÓCITOS $>12.000 \mathrm{~mm}^{3}$ ou desvio a esquerda
Quadro clínico pouco sugestivo de SEPSE (IVAS, amigdalite ou pacientes sem fatores de risco)?

\section{SIM}

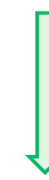

Dar seguimento ao atendimento fora do protocolo de sepse. Se alta, orientar para retornar ao hospital caso apresente sinais de deterioração.

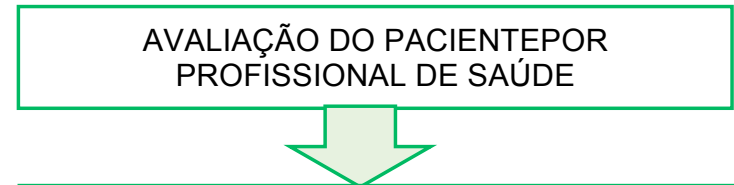

Presença de dois ou mais critérios de SIRS e/ou uma disfunção orgânica?
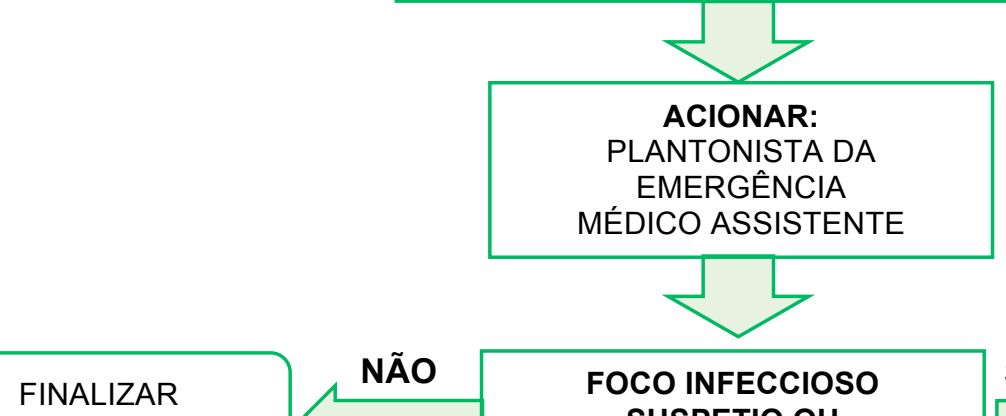
PROTOCOLO
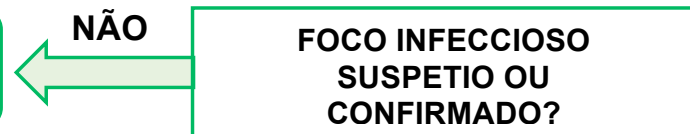

SIM

PACIENTES EM CUIDADOS

PALIATIVOS.

DISFUNÇÃO ORGÂNICA HIPOTENSÃO:

PAS $<100 \mathrm{mmHg}$, confusão, agitação ou coma;

SatO2: $<90 \%$, necessidade O2 ou dispneia;

Diurese: $<0,5 \mathrm{~mL} / \mathrm{kg} / \mathrm{hr}$; EXAMES LABORATORIAIS (CASO DISPONÍVEL)

Creatina $>0,2 \mathrm{mg} / / \mathrm{dL}$; Lactato $>2,0 \mathrm{mmol} / \mathrm{L}$ Plaquetas: $<100.000 / \mathrm{mm}^{3}$ ou RNI $>1.5$ ou TTPA > 60 seg

Bilirrubina total: $>2 \mathrm{ma} / \mathrm{dL}$
Dar seguimento ao atendimento fora do protocolo de SEPSE.

\section{NÃO}

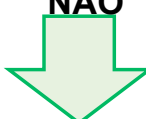

Não, Somente com SIRS

Paciente com alguma disfunção orgânica?

Redobrar a atenção - alto risco de óbito, reavaliar o paciente a cada hora.

Agilizar transferência para UTI, sempre eu possível.

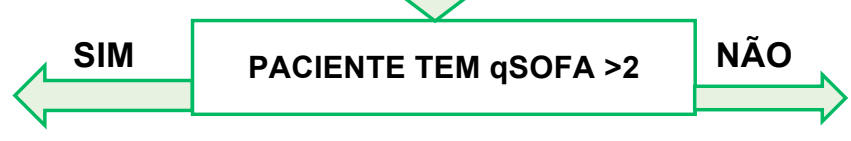

Manter cuidados habituais

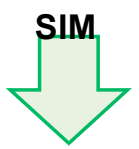

ANTIMICROBIANOS E EXAMES LABORATORIAIS

- Gasometria e lactato arterial:

- Duas culturas de sítios diferentes e de os sítios pertinentes;

Adminstrar antimicrobianos em 1 hora VALOR DE REFERÊNCIA).

3) Cristalóides $30 \mathrm{~mL} / \mathrm{kg}$ em até 3 horas

4) Vasopressores se PAS $<90 \mathrm{mmHg}$ apesar do cristaloide

6) Programar a coleta do $2^{\circ}$ lactato (se o primeiro estiver alterado) 


\section{ENFERMAGEM}

PACIENTE APRESENTA PELO MENOS UM DOS CRITÉRIOS DE DISFUNÇÃO ORGÂNICA ABAIXO?
( ) Oligúria
( ) Pressão Arterial Sistólica $<100 \mathrm{mmHg}$
( ) Rebaixamento do nível de consciência $(\mathrm{ECG}<15)$
( ) Frequência Respiratória $>22$ irpm ou dessaturação

\section{Acionamento da equipe médica}

Nome do médico chamado: Hora:

ENFERMEIRO RESPONSÁVEL: COREN:

\section{AVALIAÇÃO MÉDICA 1}

\section{PACIENTE APRESENTA HISTORIA} SUGESTIVA DE INFECÇÃO?

( ) Pneumonia/Empiema

( ) Infecção de prótese

( ) Infecção urinária

( ) Infecção óssea/articular

( ) Infecção abdominal aguda

( ) Infecção de ferida operatória

( ) Meningite

( ) Infecção de corrente sanguínea associada ao cateter

( ) Endocardite

( ) Sem foco definido

( ) Pele e partes moles

( ) Outras infecções:

\section{AVALIAÇÃO MÉDICA 2}

O PACIENTE APRESENTA CRITÉRIOS PARA:

( ) Infecção (ainda sem disfunção clínica, necessita coleta de exames para descartar disfunção

orgânica laboratorial)
( ) Sepse
( ) Afastado infecção/sepse/choque séptico
( ) Sepse /choque séptico em cuidados de fim de vida sem conduta no momento

\section{CONDUTA MÉDICA:}

( ) coletar exames do kit sepse e prescrever antimicrobiano

(Data e hora da coleta: hora:

(Data e hora da primeira dose: hora:

( ) encerrar o atendimento

Data e hora do

atendimento médico:

às ( $\ldots$ : _ $)$ 


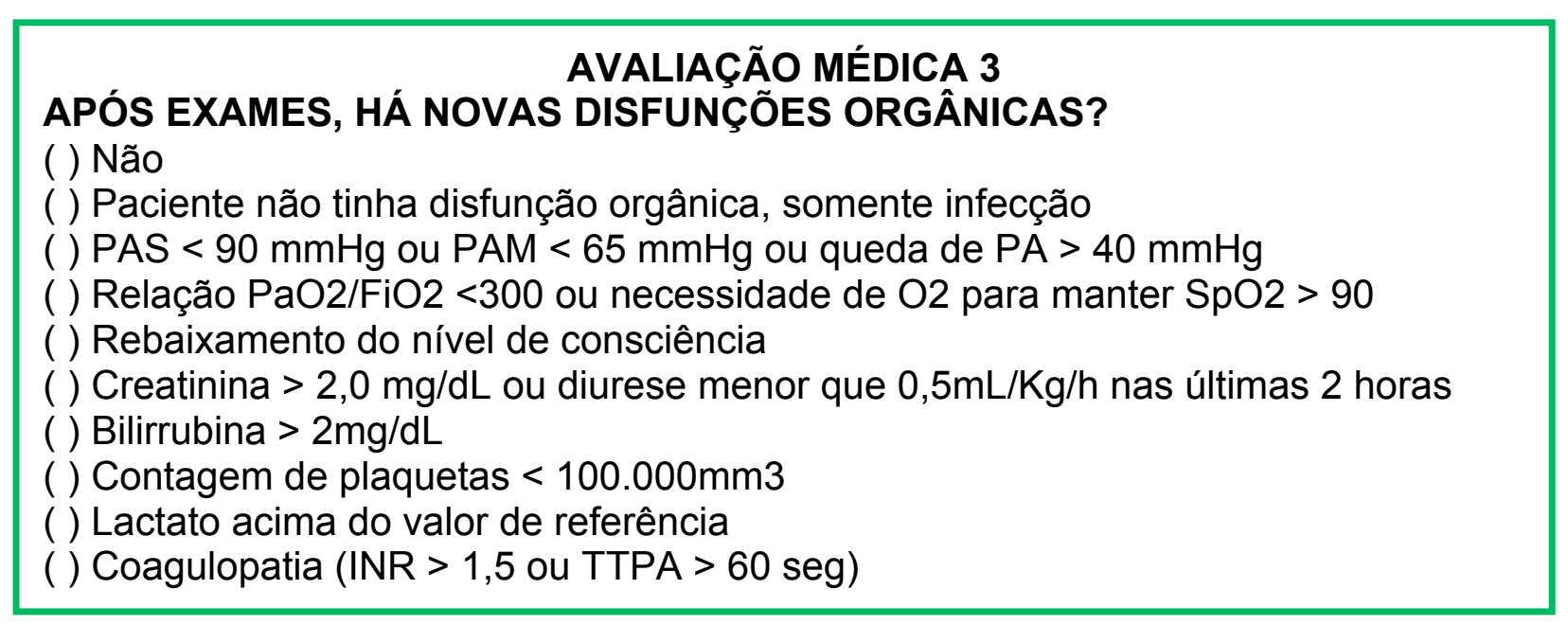

Data e hora da primeira disfunção orgânica: às

O CASO FICOU CONFIRMADO COMO:
( ) Infecção
() Sepse
( ) Sepse com lactato alterado
( ) Choque séptico
( ) Afastado infecção

MÉDICO RESPONSÁVEL: CRM: 